\title{
ELECTRICAL AND OPTICAL PROPERTIES OF ZnSe:Mg CRYSTALS
}

\author{
J. Szatkowski, F. Firszt, H. MĘCzyńska and S. ŁĘGowski
}

Institute of Physics, N. Copernicus University, Grudziądzka 5/7, 87-100 Toruń, Poland

\begin{abstract}
ZnMgSe mixed crystals were obtained by the high pressure Bridgman method and by thermal diffusion of $\mathrm{Mg}$ metal into ZnSe crystals. Measurements of luminescence and transmission spectra show that the band-gap energy of such mixed crystals is larger than that of "pure" ZnSe. ZnMgSe crystals exhibit $n$-type conductivity and electrical parameters comparable with that of $\mathrm{ZnSe}$. Blue-violet luminescence in the temperature range from $40 \mathrm{~K}$ to room temperature was observed. This feature makes this material very promising for future applications in constructing short wavelength electroluminescent devices.
\end{abstract}

PACS numbers: 78.55.Et, 71.55.Gs, 72.20.My

During last few years a significant progress has been made in constructing blue-green LED and laser diodes based on $\mathrm{ZnSe}, \mathrm{ZnCdSe}$ and $\mathrm{ZnSSe}$ layers and quantum well structures [1-4]. Recently mixed crystals of $\mathrm{Mg}$ chalcogenides with wide-gap II-VI compounds have been proposed to produce a variety of new materials for optoelectronics [5-7]. ZnMgSe mixed crystals are of special interest because they offer the possibility of obtaining blue and UV emission.

ZnMgSe crystals were grown by the high pressure Bridgman method using the Koch-Light $6 \mathrm{~N}$ ZnSe powder containing 10 and 20 mole percent of $\mathrm{Mg}$ metal as a starting material. After growing process crystals were annealed in liquid zinc or zinc vapour at temperatures $1070 \mathrm{~K}$ and $1220 \mathrm{~K}$ in order to obtain low-resistivity $n$-type material. Magnesium can be also easy incorporated into $\mathrm{ZnSe}$ by thermal annealing of undoped $\mathrm{ZnSe}$ crystals in $\mathrm{Zn}+\mathrm{Mg}$ vapour at $T=1070 \mathrm{~K}$. This process forms heavily doped $\mathrm{ZnMgSe}$ mixed crystal surface layer. Figure $1 \mathrm{~A}$ presents photoluminescence (PL) spectra of "pure" ZnSe (curve $a$ ) and ZnMgSe crystals grown from the melt with $10 \%$ (curve $b$ ) and $20 \%$ (curve $c$ ) of $\mathrm{Mg}$ in starting material. The PL spectrum of undoped ZnSe consists of weak exciton line at $2.802 \mathrm{eV}$, strong edge donor-acceptor (D-A) pair emission at $2.7 \mathrm{eV}$ with LO-phonon replicas and deep-level band at $1.95 \mathrm{eV}$. In $\mathrm{ZnMgSe}$ crystals the main shape of PL spectrum is similar to that of "pure" ZnSe but a shift of each emission band towards higher photon energies is observed indicating increase in the band-gap energy of such mixed crystals. The increase in the band-gap energy of ZnMgSe crystals with increasing $\mathrm{Mg}$ concentration was also confirmed by measurements of transmission spectra. 


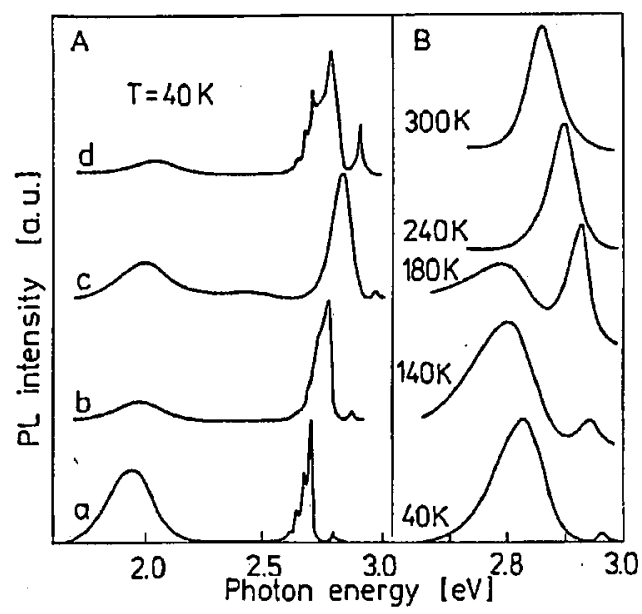

Fig. 1. Photoluminescence spectra of ZnSe and ZnMgSe crystals: (A) "pure" ZnSe curve $a, \mathrm{ZnMgSe}$ crystals grown from the melt with $10 \%$ - curve $b$ and $20 \%$ - curve $c$ of $\mathrm{Mg}$ mole fraction in starting material, $\mathrm{ZnMgSe}$ crystal obtained by thermal diffusion of $\mathrm{Mg}$ metal into "pure" $\mathrm{ZnSe}$ from $\mathrm{Zn}+\mathrm{Mg}$ vapour - curve $d$; (B) blue part of PL spectrum of $\mathrm{ZnMgSe}$ crystal grown by Bridgman method with $20 \%$ of $\mathrm{Mg}$ in starting material at different temperatures.

Comparing PL spectra of $\mathrm{ZnMgSe}$ crystals with that of "pure" $\mathrm{ZnSe}$, we conclude that emission bands with maxima at $2.76 \mathrm{eV}$ and $2.825 \mathrm{eV}$ (Fig. 1A, curves $b$ and $c$ ) correspond to edge emission in $\mathrm{ZnMgSe}$ mixed crystals with different $\mathrm{Mg}$ concentrations. The lines with maxima at $2.87 \mathrm{eV}$ and $2.96 \mathrm{eV}$ (Fig. 1A, curve $b$ and $c$ ) are believed to be of excitonic origin. Figure $1 \mathrm{~A}$ (curve $d$ ) presents the PL spectrum of $\mathrm{ZnSe}$ crystal into which $\mathrm{Mg}$ was diffused from $\mathrm{Zn}+\mathrm{Mg}$ vapour. The heavily doped surface layer was partially etched before PL measurements. In PL spectrum of these crystals, except from the mentioned above exciton $(2.91 \mathrm{eV})$ and edge $(2.79 \mathrm{eV})$ emission from mixed crystals the edge emission from "pure" ZnSe at $2.7 \mathrm{eV}$ is also observed, probably due to inhomogeneous doping of the surface layer with $\mathrm{Mg}$. It should be noted here that the ratio of blue emission intensity to that of deep levels is greater for $\mathrm{ZnMgSe}$ than for "pure" ZnSe. Figure 1B presents blue part of PL spectra at different temperatures from $40 \mathrm{~K}$ to room temperature (RT) of $\mathrm{ZnMgSe}$ crystal grown from the melt with $20 \%$ of $\mathrm{Mg}$ in starting material. The edge emission is thermally quenched at temperature $200 \mathrm{~K}$ and only one blue band at $2.85 \mathrm{eV}$ is observed at room temperature. The blue PL band at $\mathrm{RT}$ is observed in all $\mathrm{ZnMgSe}$ crystals after annealing in liquid zinc. As grown mixed crystals do not exhibit any blue luminescence at RT. At $T=40 \mathrm{~K}$ the intensity of blue emission in these crystals is very low probably due to creation of large number of cation vacancies during high temperature growing process, which produce strong deep-level emission at about $2.0 \mathrm{eV}$ and cause very high electrical resistivity of such a material. Annealing of $\mathrm{ZnMgSe}$ crystals in liquid $\mathrm{Zn}$ leads to 


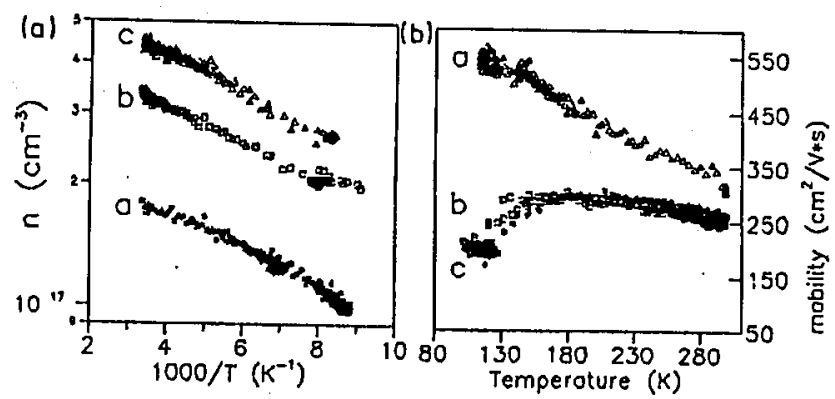

Fig. 2. Temperature dependence of electron concentration (a) and Hall mobility (b) in: $a-\mathrm{ZnSe}$ crystal, $b$ and $c-\mathrm{ZnMgSe}$ crystals annealed in liquid zinc and $\mathrm{Zn}+\mathrm{Mg}$ vapour at $T=1070 \mathrm{~K}$, respectively.

$n$-type conductivity with resistivity less than $0.5 \Omega \mathrm{cm}$. Electrical properties of such crystals were measured by the Van der Pauw method.

Figure 2 shows the electron concentration and the Hall mobility as a function of temperature for "pure" $\mathrm{ZnSe}(a)$ and for $\mathrm{ZnMgSe}$ crystals grown from the melt with $10 \%$ of $\mathrm{Mg}$ metal in starting material and then annealed in liquid zinc and $\mathrm{Zn}+\mathrm{Mg}$ vapour $(b, c)$. The electron concentration is larger in $\mathrm{ZnMgSe}$ crystal than in "pure" $\mathrm{ZnSe}$ which indicates that the concentration of isolated zinc vacancies or zinc vacancies complexes, which act as deep compensating acceptors, is lower in mixed crystals than in $\mathrm{ZnSe}$. Using nondegenerate statistics, we obtained an activation energy about $10 \mathrm{meV}$ for electrons in "pure" ZnSe and similar value for $\mathrm{ZnMgSe}$ crystals. This value is considerably smaller than the activation energy of $28 \mathrm{meV}$ for isolated III-group donors in ZnSe derived from optical spectroscopy [8]. The small value observed for the activation energy in the temperature dependence of the free-carrier concentration is therefore a strong indication that $n$-type ZnMgSe crystals are in large degree compensated. From the study of the Hall data, various possible scattering mechanisms were considered within the theoretical analysis presented by Ruda [9]. The Hall mobility for undoped ZnSe crystal increases monotonically with decreasing temperature in the range $100-300 \mathrm{~K}$, indicating that the major scattering mechanism is the scattering by optical phonons. The temperature dependence of mobility in $\mathrm{ZnMgSe}$ crystals indicates that the scattering by charged impurities plays an important role at temperatures lower than $140 \mathrm{~K}$. The effect of the hydrostatic pressure on electron concentration and the Hall mobility was also measured but we did not observe any significant changes of electrical parameters with pressure up to $10 \mathrm{kbar}$.

To our knowledge $\mathrm{ZnMgSe}$ mixed crystals grown from the melt have not been investigated previously. Our results (low electrical resistivity and blue PL observed up to room temperature) indicate that this material can be very promising for future applications in constructing blue-violet electroluminescent devices. 


\section{References}

[1] X.W. Fan, J. Woods, IEEE Trans. Electron. Devices ED28, 428 (1981).

[2] M.A. Haase, J. Qiu, J.M. DePuydt, H. Cheng, Appl. Phys. Lett. 59, 1272 (1991).

[3] A.W. Livingstone, K. Turvey, J.W. Allen, Solid State Electron. 16, 351 (1973).

[4] W. Xie, D.C. Grillo, R.L. Gunshor, M. Kobayashi, H. Jeon, J. Ding, A.V. Nurmiko, G.C. Hua, N. Otsuka, Appl. Phys. Lett. 60, 1999 (1992).

[5] H.J. Łożykowski, P.O. Holtz, B. Monemar, J. Electron. Mater. 12, 653 (1983).

[6] H. Okuyama, K. Nakano, T. Miyajima, K. Akimoto, Jpn. J. Appl Phys. 30, L1620 (1991).

[7] Y. Morinaga, H. Okuyama. K. Akimoto, Jpn. J. Appl. Phys. 32, 678 (1993).

[8] W. Stutius, J. Appl.Phys. 53, 284 (1982).

[9] H.E. Ruda, J. Appl. Phys. 59, 1220 (1986). 